\title{
Surface Enhanced Raman Spectroscopy for Molecular Identification- a Review on Surface Plasmon Resonance (SPR) and Localised Surface Plasmon Resonance (LSPR) in Optical Nanobiosensing
}

Leda G. Bousiakou, ${ }^{1, *}$ Hrvoje Gebavi, ${ }^{2}$ Lara Mikac, ${ }^{2}$ Stefanos Karapetis, ${ }^{3}$ Mile Ivanda ${ }^{2}$

\footnotetext{
1 IMD Laboratories Co, R\&D Section, Lefkippos Technology Park, NCSR Demokritos PO Box 60037, GR-15130 Agia Paraskeyi, Athens, Greece

2 Center of Excellence for Advanced Materials and Sensing Devices, Ruđer Bošković Institute, Bijenička cesta 54, HR-10000 Zagreb, Croatia

3 Laboratory of Inorganic \& Analytical Chemistry, School of Chemical Engineering, Dept of Chemical Sciences, National Technical University of Athens, 9 Iroon Polytechniou St., GR-15780, Athens, Greece

* Corresponding author's e-mail address: leda@imdlaboratories.g
}

RECEIVED: August 4, 2019 * REVISED: October 20, 2019 « ACCEPTED: October 20, 2019

\begin{abstract}
Surface plasmon resonance (SPR) allows for real-time, label-free optical detection of many chemical and biological substances. Having emerged in the last two decades, it is a widely used technique due to its non-invasive nature, allowing for the ultra-sensitive detection of a number of analytes. This review article discusses the principles, providing examples and illustrating the utility of SPR within the frame of plasmonic nanobiosensing, while making comparisons with its successor, namely localized surface plasmon resonance (LSPR). In particular LSPR utilizes both metal nanoparticle arrays and single nanoparticles, as compared to a continuous film of gold as used in traditional SPR. LSPR, utilizes metal nanoparticle arrays or single nanoparticles that have smaller sizes than the wavelength of the incident light, measuring small changes in the wavelength of the absorbance position, rather than the angle as in SPR. We introduce LSPR nanobiosensing by describing the initial experiments performed, shift-enhancement methods, exploitation of the short electromagnetic field decay length, and single nanoparticle sensors are as pathways to further exploit the strengths of LSPR nanobiosensing. Coupling molecular identification to LSPR spectroscopy is also explored and thus examples from surface-enhanced Raman spectroscopy are provided. The unique characteristics of LSPR nanobiosensing are emphasized and the challenges using LSPR nanobiosensors for detection of biomolecules as a biomarker are discussed.
\end{abstract}

Keywords: optical nanobiosensors, plasmonic, surface plasmon resonance, SPR, Raman, LSPR

\section{INTRODUCTION}

$\mathbf{T}$ HE advancement of nanoscale materials over the last two decades has led to the introduction of nanobiosensors offering highly sensitive detection at the single molecular level as well as enabling the advancement of point of care diagnostics. ${ }^{[1-7]}$ In particular optical nanobiosensors are at the forefront of research measuring both catalytic and affinity reactions by utilizing a number of optical transduction routes, ${ }^{[2]}$ such as absorption, fluorescence, phosphorescence, Raman, surface enhanced Raman spectroscopy (SERS) and dispersion spectroscopy measuring a number of property changes such as energy, polarization, decay time, phase and amplitude. Additionally, due to their non-electrical nature optical nanobiosensors are the candidates of choice for in vivo applications enabling multiple target detection by measuring different wavelengths. The basic operational principle of optical nanobiosensors includes the immobilization of bioreceptor molecules in direct spatial coupling to the transducer either by physical entrapment or chemical attachment converting the binding event into a measurable optical signal. The use of optical fiber probes in biosensing is a popular route based on the absorption, fluorescence or light scattering, reporting on wavelength changes, wave propagation, time, intensity, spectrum distribution or light

((c) $\mathrm{EY}$ This work is licensed under a Creative Commons Attribution 4.0 International License. 

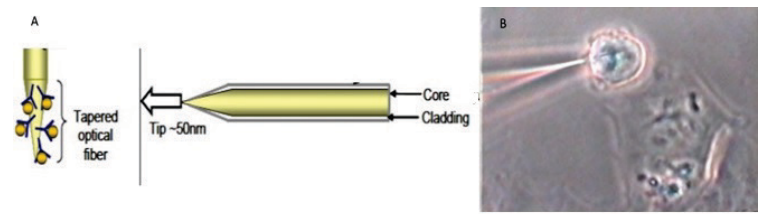

Figure 1. (A) A schematic representation of an optical nanoimmunosensor; ${ }^{[9]}$ (B) An optical nanosensor developed by Vo-Dinh's group to study cell function and drug effectiveness. In this image it probes a single living cell. ${ }^{[10]}$

polarity. ${ }^{[8]}$ There is a sche-matic representation of an antibody based optical nanobiosensor able to perform qualitative and quantitative measurements in single living cells (Figure 1).

Currently, the most common transducers ${ }^{[2]}$ utilized in optical nanobiosensing are fluorescence spectroscopy, surface plasmon resonance, interferometry and spectroscopy of guided modes in optical waveguide structures. Notable examples include fluorescence resonance energy transfer (FRET) that utilizes quantum dots (QDs) as part of nanoassemblies that operate based on electronic excitation energy flow. ${ }^{[11,12]}$ In particular QDs (donors) absorb light energy and transfer it to an acceptor such as an organic fluorophore resulting in the donor's fluorescence quenching which can be easily observed (Figure 2).

Furthermore, advances in local surface plasmon resonance (LSPR) using metallic nanoparticles has allowed for high precision measurements at the molecular level that can be readily combined with SERS studies offering enhanced Raman signals for precise molecular identification. In particular, the use of SERS nanoprobes for single molecule detection for the imagining of cell surface receptors results in significant spatial and spectral information. ${ }^{[14-18]}$ In the following sections we shall focus on plasmonic biosensing and in particular look at the principles of surface plasmon resonance (SPR). Furthermore, we shall discuss the scientific background of local surface plasmon resonance (LSPR) within nanobiosensing and make a comparison with SPR. Finally, SERS shall be discussed due to its ability in molecular identification using plasmonic nanoparticles.

\section{SURFACE PLASMON RESONANCE (SPR)}

Liedberg et al in $1983^{[19]}$ demonstrated surface plasmon resonance (SPR) for biosensing applications using the Kretschmann configuration. ${ }^{[20]}$ In particular by detecting real time small changes in the refractive index at the interface between a thin metal film and a dielectric fluid upon binding of a target species, they were able to provide a label-free, non-invasive biosensing mechanism based on SPR. Any mass increase on the biosensing surface led to a proportional increase in the refractive index which was observed as a shift in the resonance angle. In general, if a light beam propagates between two non-absorbing media where $n_{1}>n_{2}$ at an angle of incidence above a critical angle then the light is totally internally reflected (TIRE) at the interface, propagating back into the high refractive index medium. Nevertheless, still an evanescent field $\left(k_{\mathrm{x}}\right)$ will penetrate the exit medium. If the interface of the TIRE is coated with a thin layer of suitable material (e.g. noble metal) and p-polarized light is shone on the interface, then the evanescent wave penetrating the metal layer will excite electromagnetic surface plasmon waves $\left(k_{\mathrm{SP}}\right)$, ie. collective electron oscillations.[22-26] In the condition of surface plasmon resonance (SPR), $k_{x}$ should be equal to $k_{\mathrm{SP}}\left(k_{\mathrm{x}}=k_{\mathrm{SP}}\right)$ and the photon momentum is equal to the plasmon momentum. This can be achieved using adaptive optics (Figure 3).

Thus, for a certain angle of incidence in a Kretschmann configuration, $\mathrm{p}$-polarized light could lead to surface plasmon resonance (SPR). This can be accomplished by scanning the angle of incidence of light at a fixed wavelength or by using a broad light source with multiple wavelengths. Once the resonant conditions are met a dip in the reflectivity of light is experienced, which would not have been possible under s-polarization conditions (Figure $4 \mathrm{~A})$. Considering that the resonant condition is sensitive to the nature of the interface the absorbance of a thin layer of biomolecules will lead to a shift in SPR (Figure 4B).

It is important to note that surface plasmons in such as set up act as antennas in sensing the presence of the analytes in solutions up to a distance of the evanescent

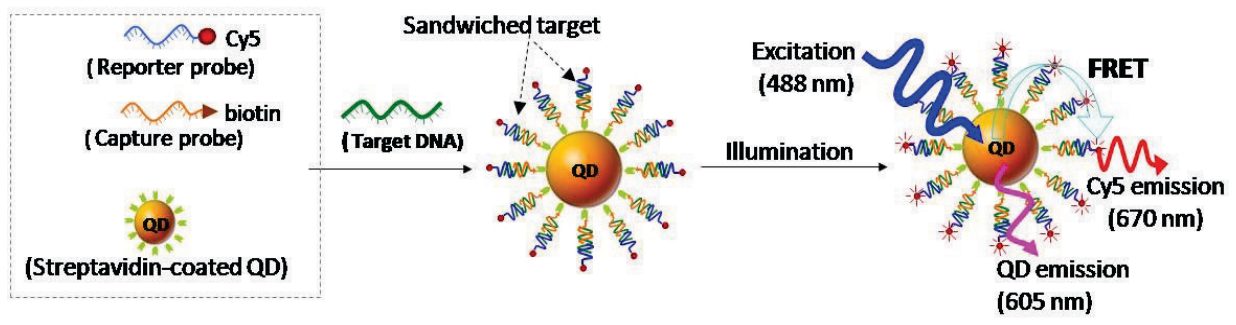

Figure 2. Principle of quantum dots employed in FRET nanobiosensing for DNA detection. ${ }^{[13]}$ 

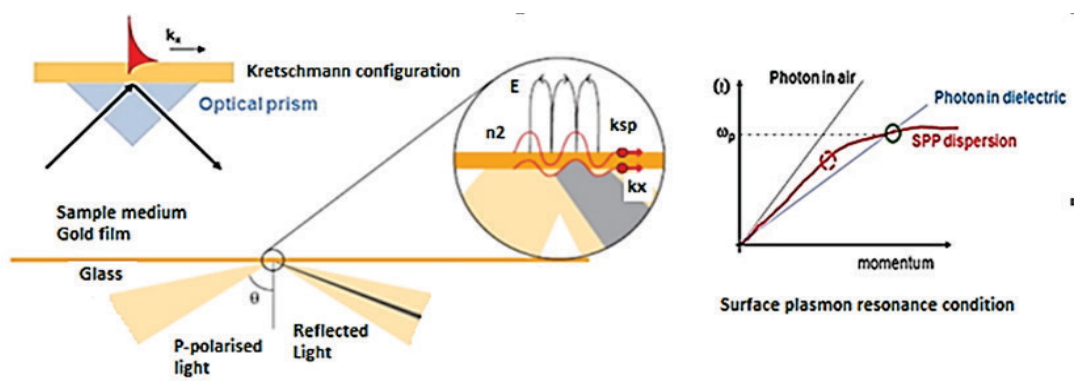

Surface plasmon resonance condition

Figure 3. A schematic representation of SPR in the Kretschmann configuration. ${ }^{[21,27]}$

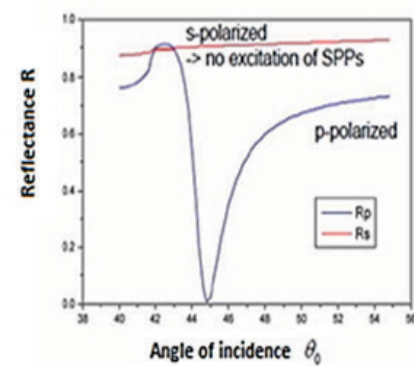

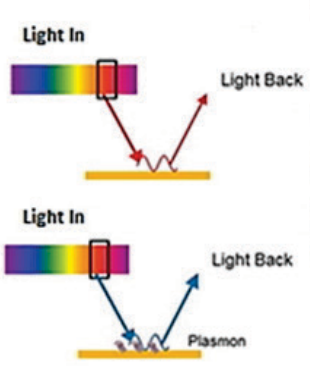

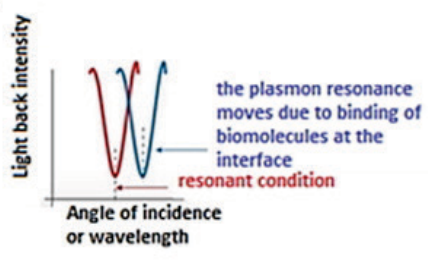

Figure 4. (A) Dip in reflectivity under plasmon resonance conditions; (B) Plasmon resonance shift. ${ }^{[28]}$

field in the dielectric denoted as $\delta_{\text {diel }}$ (Figure 5). In particular as seen:

- $\delta_{\text {diel }}$ is the evanescent field length in the dielectric representing how far in the dielectric the surface plasmon wave extends

- $\delta_{\text {metal }}$ is the evanescent field length in the metal representing how far into the metal the surface plasmon wave reaches

- $\delta_{\text {SPP }}$ is the surface plasmon propagation distance representing how far from the excitation point the surface plasmon can travel along the metal/dielectric interface.

Some typical orders of magnitude of the plasmon lengths for an excitation light of $\lambda=500 \mathrm{~nm}$, are $\delta_{\text {diel }}=$ $250-1000 \mathrm{~nm}, \delta_{\text {metal }}=5 \mathrm{~nm}$ and $\delta_{\mathrm{SPP}}=2-20 \mu \mathrm{m}$, while at $\lambda=$ $1500 \mathrm{~nm}, \delta_{\mathrm{SPP}}$ can be up to $1 \mathrm{~mm}$. It is thus evident that even if the penetration in the metal is small, the plasmon can sense up to a micron away from the metal surface. In general, the preferred metal for thin film deposition in SPR

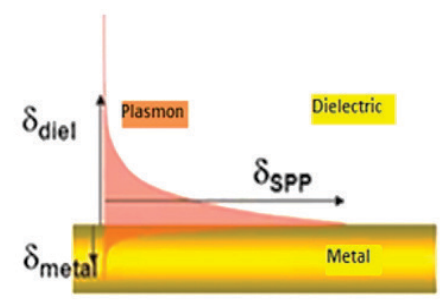

Figure 5. Surface plasmons are characterized by three length scales $\delta_{\text {diel, }} \delta_{\text {metal }}$ and $\delta_{\text {Spp. }}{ }^{[28]}$ is gold (approx. $50 \mathrm{~nm}$ ) as it can facilitate with ease a strong and easy to measure SPR signal in the near infrared region, while keeping resistant to oxidation and other atmospheric contaminants. Furthermore, it is easy to functionalize showing compatibility with a variety of chemical modification systems.

\section{Localized Surface Plasmon Resonance (LSPR) and Nanobiosensing}

Despite the fact that surface plasmon resonance (SPR) is achieved when the metal thin film layers are in the $\mathrm{nm}$ range, it is localized surface plasmon resonance (LSPR) that takes us further into nanobiosensing offering unique opportunities not only in signal specificity and accuracy but also in sensor size. The principles of biosensing in both
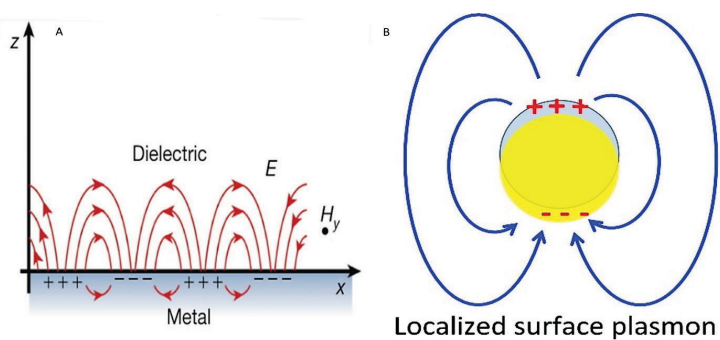

Figure 6. (A) Thin metal film the surface plasmon (polariton) propagates parallel to the negative permittivity/dielectric material interface (B) surface plasmon confined at the surface of the plasmonic nanostructures. ${ }^{[29,30]}$ 
cases are based on the creation of surface plasmons on metallic surfaces and the relative changes of refractive index upon target analyte attachment. Nevertheless, in the case of plasmonic nanobiosensing the role of metallic nanoparticles (NPs) and periodic nanoarrays becomes central; in particular in bounded geometries when the lateral dimensions of interface become much smaller than $\delta_{\text {spp }}$ surface plasmons become localized (Figure 6).

In general, localized surface plasmon resonance (LSPR) is an optical phenomenon resulting from the interaction of light with conductive nanoparticles whose size is smaller than the incident wavelength. More specific the electric field of the incident light leads to collective excitations of the electrons in the conduction band resulting in 'coherent localized plasmon oscillations with a resonant frequency depending on the composition, size, geometry, dielectric environment and separation distance of the $N P s^{\prime}{ }^{\left[{ }^{[31-33]}\right.}$ Resonance in LSPR can be achieved without the need for adaptive optics as in SPR. In particular if consider a nanoparticle in terms of a negatively charged electron cloud around a positively charged static ionic core then resonance can be induced by selecting the appropriate

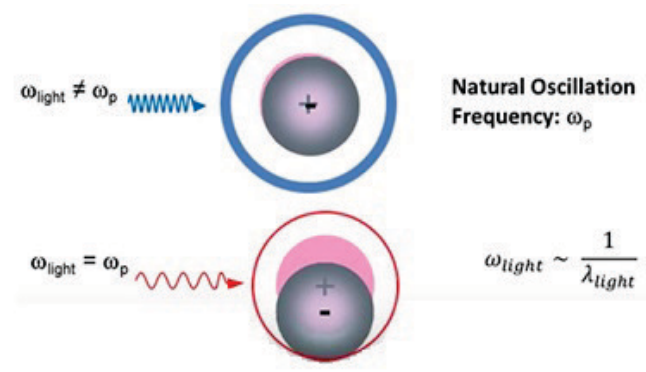

Figure 7. If we perturb the nanoparticle which has a natural oscillation frequency of $\omega_{p}$ by light of the same frequency, the incident light is absorbed by the oscillation and thus enhancing its amplitude with little light scattered. ${ }^{[28]}$
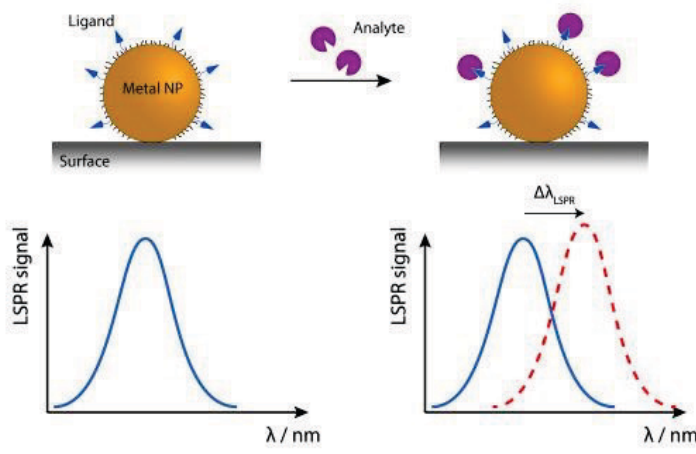

Figure 8. Observation of upon binding of the analyte with the ligand on the nanoparticle surface. ${ }^{[34]}$ wavelength of light such that the natural oscillation frequency of the nanoparticle $\omega p$ equals the one of the perturbing light, i.e. wlight (Figure 7).

In the schematic representation (Figure 8) the basic concept behind nanoplasmonic sensing is displayed; initially when gold nanoparticles are illuminated strong absorption and scattering occurs leading to the extinction of the incident light with a characteristic peaking spectral distribution. Upon interaction of the analyte with a ligand on the surface of the metallic nanoparticle a shift of the plasmon peak position in the extinction spectra is observed.

In general, as most LSPR methods involve ensembles of nanoparticles that can have different sizes, the signals obtained are average signals and can exhibit heterogeneous broadening (Figure 9), compared to the signal from each individual resonance of a single nanoparticle.

\section{A Comparison of SPR and LSPR: Advantages and Disadvantages}

Comparing surface plasmon resonance (SPR) and localized surface plasmon resonance (LSPR) biosensing we note that the resonance conditions in LSPR can be achieved without the need for adaptive optics as in SPR thus reducing engineering challenges. Furthermore, in SPR thermal control is often required in order to maintain the incidence angle of light within 0.001 of a degree. In contrast the LSPS can be coupled directly to the incident light leading to simpler detection systems. Moreover, the evanescent field of a localized surface plasmon is much shorter $\left(\delta_{\text {diel }}\right.$ is approximately $20-40 \mathrm{~nm}$ ) that the evanescent field of a surface plasmon. This means that LSPR senses only at a distance of a few $\mathrm{nm}$, while SPR senses at a distance of up to a micron. The difference in the sensing volume of the respective plasma is referred to as the bulk effect (Figure 10). In particular, due to the much larger field of the SPR which goes up to $1000 \mathrm{~nm}$ versus the LSPR's $40 \mathrm{~nm}$,

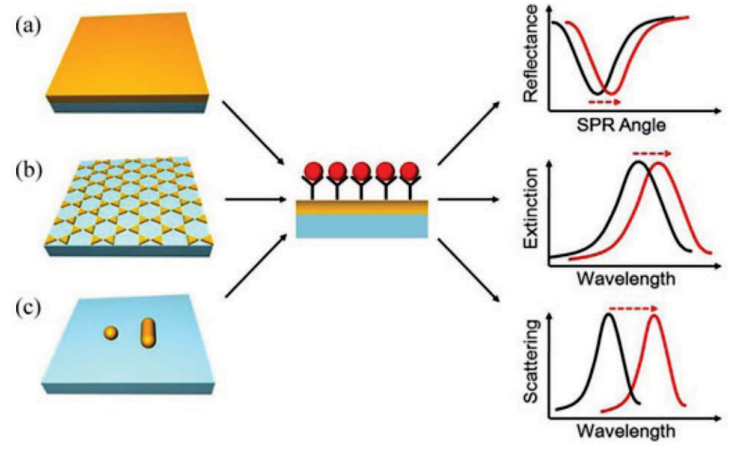

Figure 9. Optical signals in the case of (a) metal thin film biosensors (SPR), (b) LSPR nanobiosensing using arrays of nanoparticles (c) single metallic nanoparticle biosensing. ${ }^{[35]}$ 
biomolecules that are not actually bound at the sensors surface can be detected in SPR causing a bulk effect or false positive reading. In contrast in LSPR only the molecules bound at the surface are captured by the surface localized plasmons. The others are invisible to the sensor and do not contribute to the signal. Thus, LSPR has a marginal bulk effect. In fact, the large evanescent field of SPR is responsible for the large nonspecific bulk effect and incontrollable signal fluctuations which generally makes it incompatible with complex matrices. Thus, SPR has not been successfully applied to the diagnostic world particularly for blood-based immunoassays where detection of low-level biomarkers requires signal amplification.

LSPR sensors are capable in detecting small molecules (molecular weight $<1000 \mathrm{~g} \mathrm{~mol}^{-1}$ ) as well as being able to detect very low amounts of the target analyte with current research aiming at single molecule detection at in vivo applications, d. LSPR offers excellent tunability in

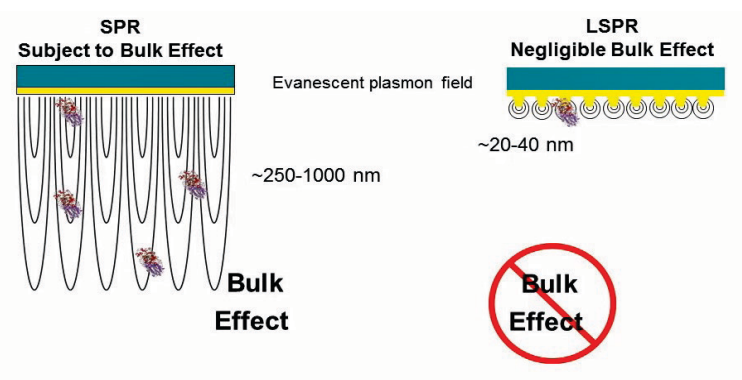

Figure 10. The bulk effect in SPR biosensing compared to LSP (SPR). ${ }^{[28]}$ sensing by altering nanoparticle shapes, sizes and composition. In Table 1, a comparison between SPR and LSPR sensors is displayed.

LSPR can be very well combined with SERS for molecular identification purposes. ${ }^{[37-39]}$ In general, the main limitations of the classical Raman spectroscopy are related to molecules with very low Raman efficiency, samples at very low concentration (traces) and small quantities/volumes of samples. Nevertheless, the Raman scattering intensity coming from molecules located in the vicinity of structured metallic nanostructured surfaces excited by visible light can be strongly enhanced by an order of up to 1010 . This phenomenon, known as surface enhanced Raman spectroscopy (SERS) and has become an important analytical tool molecular identification based on vibrational fingerprints. It should be noted that the SERS spectrum of a molecule might not be very similar to its classical Raman spectrum with the majority of vibrational bands corresponding to the vibrations of the closest bonds

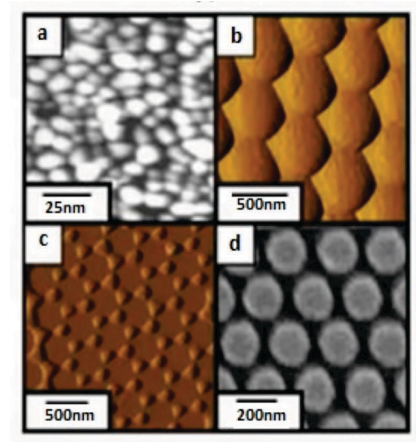

Figure 11. Different SERS active substrates. ${ }^{[40]}$

Table 1. Comparison of SPR and LSPR sensors. ${ }^{[36]}$

\begin{tabular}{|c|c|c|}
\hline Feature / characteristic & SPR & LSPR \\
\hline Label-free detection & Yes & Yes \\
\hline Distance dependence & $\approx 1000 \mathrm{~nm}$ & $\approx 30 \mathrm{~nm}$ (size tunable) \\
\hline Refractive index sensitivity & $2 \times 10^{6} \mathrm{~nm} \mathrm{RIU}{ }^{-1}$ & $2 \times 10^{2} \mathrm{~nm} \mathrm{RIU}{ }^{-1}$ \\
\hline Modes & Angle shift, wavelength shift, imaging & Extinction, scattering, imaging \\
\hline Temperature control & Yes & $\mathrm{N}$ \\
\hline Chemical identification & SPR-Raman & LSPR-SERS \\
\hline Cost & US $\$ 150,000-300,000$ & $\begin{array}{l}\text { US } \$ 5,000 \text { (multiple particles) } \\
\text { US } \$ 50,000 \text { (single nanoparticle) }\end{array}$ \\
\hline Spatial resolution & $\approx 10 \times 10^{10} \mu \mathrm{m}$ & 1 nanoparticle \\
\hline Nonspecific binding & $\begin{array}{l}\text { Minimal (determined by surface chemistry } \\
\text { and rinsing) }\end{array}$ & $\begin{array}{c}\text { Minimal (determined by surface chemistry } \\
\text { and rinsing) }\end{array}$ \\
\hline Real-time detection & Time scale $=10^{-1}-10^{3} \mathrm{~s}$, planar diffusion & Time scale $=10^{-1}-10^{3} \mathrm{~s}$, radial diffusion \\
\hline Multiplexed capabilities & Yes & Yes-possible \\
\hline Small molecule sensitivity & Good & Better \\
\hline Microfluids compatibility & Yes & Possible \\
\hline
\end{tabular}


to the metallic surface. In general, the enhancement of the Raman signal depends on the shape and size of the nanostructures. Common SERS substrates include gold/or silver nanoparticles, nanoscale roughened surface onto which the sample is adsorbed and colloid suspensions (Figure 11). There are different types of SERS substrates and in particular metal island films, metal films over nanospheres, triangular nanoparticle arrays fabricated by nanosphere lithography (NSL) and cylindrical nanoparticle arrays fabricated by electron beam lithography (EBL). Generally, if the nanoparticle network is uniform (Same size, shape and interparticle spacing) the enhancement will be uniform= uniform/reproducible SERS signal.[40]

The two main mechanisms involved in enhancing the Raman signal are the long-range electromagnetic effects due to optical properties of nanostructured metallic surfaces- LSPR (Figure 12) ( 108 enhancement) and the shortrange chemical effect due to the charge transfer between chemisorbed species and the metal surface $\left(\sim 10^{2}\right.$ enhancement).

The interaction of a metallic particle with a light leads to the creation of a local field around it which is

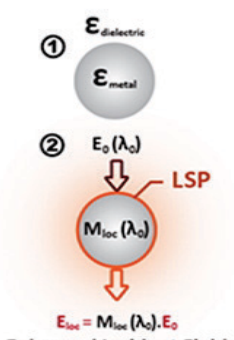

Enhanced Incident Field
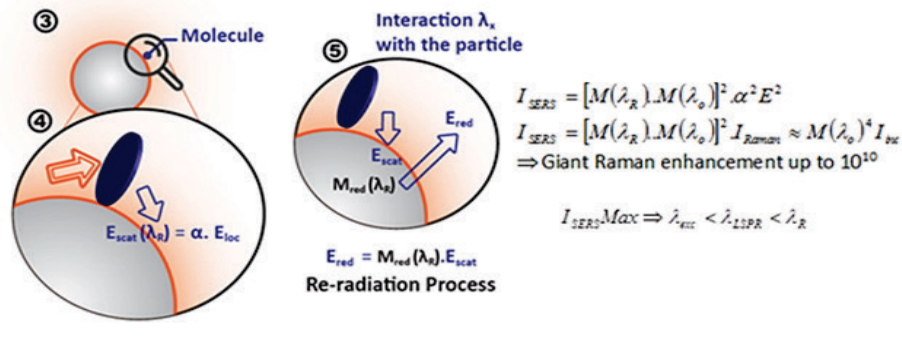

Figure 12. Raman signal enhancement due to LSPR. ${ }^{[41]}$
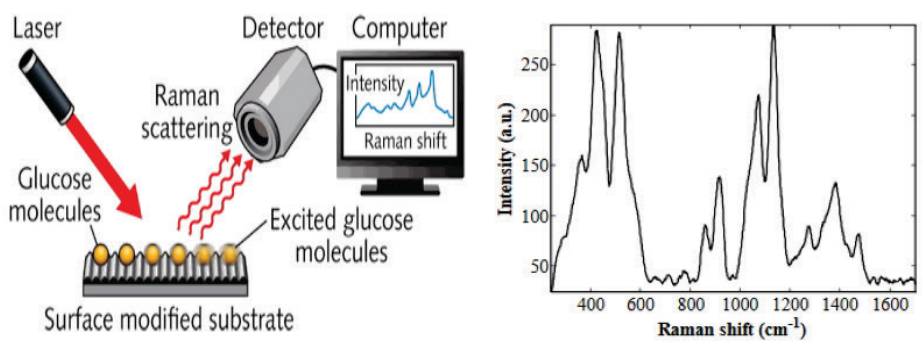

Figure 13. Glucose detection using SERS and the corresponding Raman spectrum showing scattered intensity versus energy shift for glucose. ${ }^{[42]}$
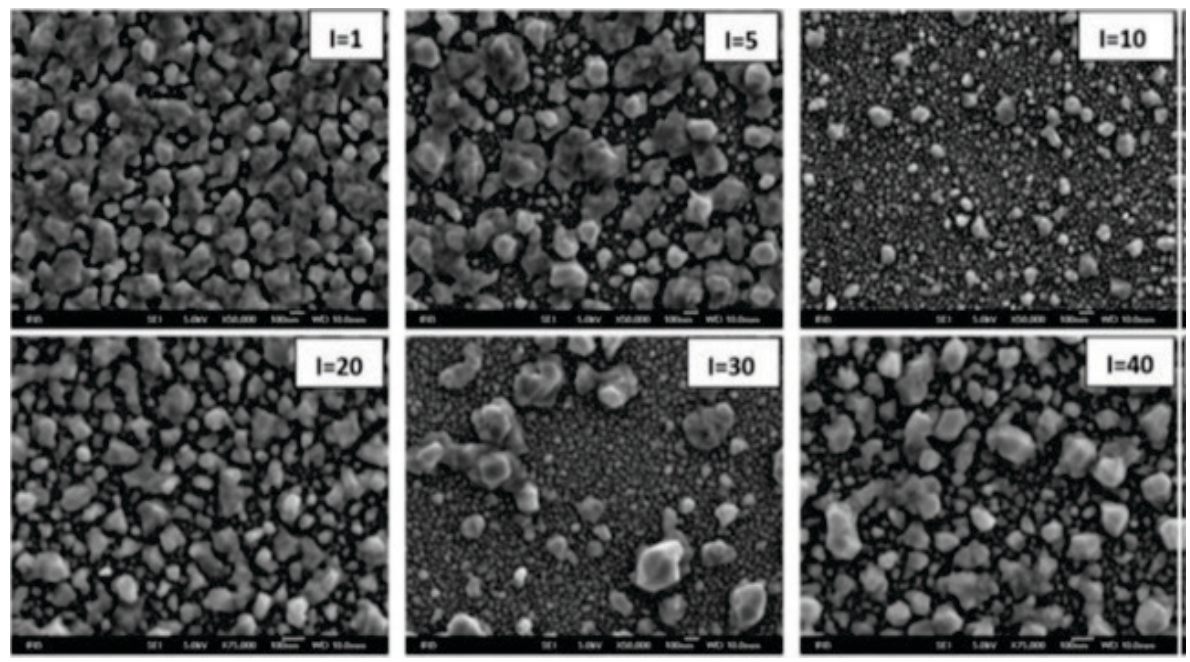

Figure 14. SEM images of Ag NPs obtained by immersion of mesoporous Si for 1 min in $10^{-2} \mathrm{M} \mathrm{AgNO}_{3}{ }^{[43]}$ 
proportional to the incident excitation. Once a molecule is in the vicinity of such a nanoparticle it will be polarized as a result of the local field and Raman scattering will occur. The scattered field in the Raman signal interact with the nanoparticle resulting an enhanced signal. This $f$ enhancement will strongly depend on the incident excitation wavelength and the observed Raman scattering, i.e. collection and detection of the signal depending on the optical numerical aperture, e.g. objective lens. In Figure 13 an optical nanobiosensor is depicted for use in glucose detection utilizing an etched silicon substrate to form nanogaps and then coated with thin $\mathrm{Au}$ and $\mathrm{Ag}$ layers leading to enhanced Raman signals (SERS).

For example, Mikac et.al..[3] prepared a series of mesoporous Si samples by changing the anodization current density. The immersion plating method was used to coat mesoporous and crystal Si samples with silver (Figure 14). By carefully choosing the anodisation parameters, they successfully influenced the size of silver crystals formed on mesoporous silicon and directly affected SERS enhancement.

The same group successfully synthesized a range of silver colloids, prepared by reducing silver nitrate with citrate, ascorbic acid, $\mathrm{NaBH}_{4}, \mathrm{PVP}$, and glucose in the presence of stabilizing agents. ${ }^{[44]}$ The results confirmed that stable silver nanoparticles of a certain size and charge were formed. The Raman enhancement was tested on pyridine and R6G and the best enhancement for pyridine was achieved using silver nanoparticles of $40 \mathrm{~nm}$ size reduced and stabilized with citrate. The SERS signal of analyte molecules was further enhanced with the addition of sodium borohydride as an alternative aggregating agent. The SERS enhancement factors for pyridine were calculated taking into account the number of molecules on the silver nanoparticles in laser activation volume and for the best results was estimated to be of the order $10^{6}$. These colloidal SERS substrates were used for the detection of histamine in fresh fish. ${ }^{[45,46]}$ Histamine is a biogenic amine responsible for majority of health problems associated with seafood consumption. The measurement parameters for detection of histamine using silver colloid SERS substrates were optimized, enabling construction of calibration curve and detection of histamine in solution at concentration of $5 \times 10^{-6} \mathrm{~mol} \mathrm{~L}^{-1}$. Conducted chemometric analysis (PCA plot and PLSR models) confirmed reliability in histamine concentration range $\left(0-200 \mathrm{mg} \mathrm{kg}^{-1}\right)$.

\section{SPR and LSPR Instrumentation: a Comparison of Fabrication Designs}

As already discussed, local surface plasmon resonance (LSPR) is instrumentally less complex than surface plasmon resonance (SPR) which requires adaptive optics (e.g. Kretschmann configuration) as well as thermal controls in order to maintain the incidence angle within 0.001 of a degree. Furthermore, in SPR the reflected light intensity is measured with a detector which must be precisely aligned in order to obtain the required signals. The first SPR biosensors were developed by Pharmacia in 1984 leading to the first commercial Biacore SPR biosensor sold in 1990. ${ }^{[47,48]}$ Since then a variety of designs have been available commercially as seen in Figure 15 below where a schematic representation of the SPR operation principle is displayed along with a contemporary Biacore X100 design by GE that offers biosensing including kinetic, affinity, specificity and concentration analysis in real time.

Biacore X100 uses Au thin film chips (Figure 16 A).Those Chips attached with carboxymethylated dextran covalently to their surface allowing molecule immobillization through $-\mathrm{NH}_{2},-\mathrm{SH},-\mathrm{CHO},-\mathrm{OH}$ and $-\mathrm{COOH}$ groups. Once the target analyte is bound to the chip surface the response is proportional to the bound mass with sensitivity of up to a few picograms (Figure $16 \mathrm{~B}$ ).

In contrast due to the LSPR resonance conditions being much simpler, the LSPR instrumentation can consist of a white light source such as room light or white LEDs along with a detector such as spectrometer, CCD camera or photodiode without the need for any thermal controls. Below a laboratory bench LSPR sensor is depicted (Figure 17)
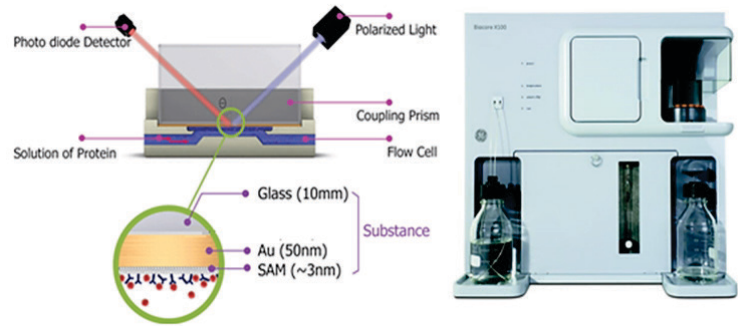

Figure 15. SPR operating principle of Biacore X100.[49]

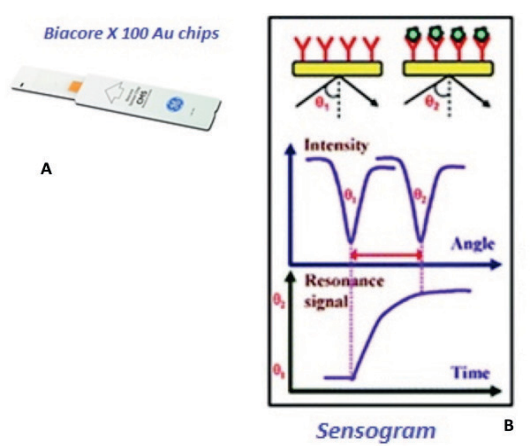

Figure 16. (A) Biacore X100 Au chips; (B) SPR sensogram. [50] 


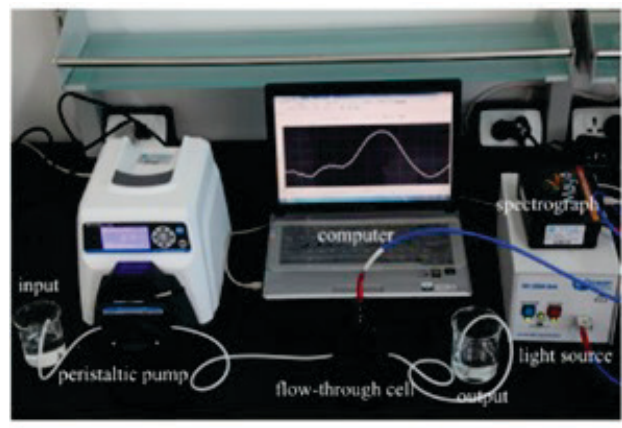

(a)

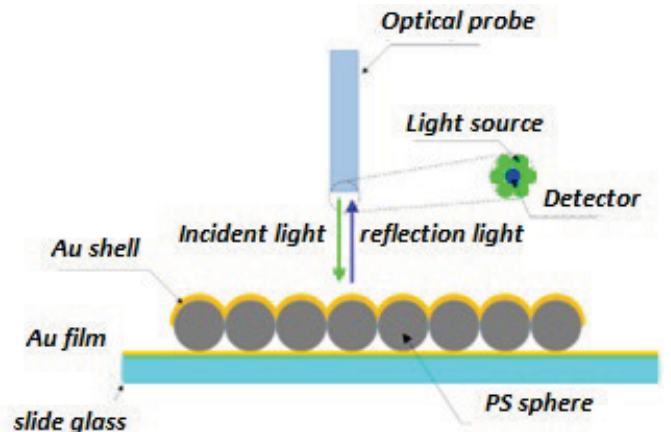

(b)

Figure 17. (a) The LSPR detection system (b) A schematic diagram of its operational principle based on core-shell nanosensors (spherical polystyrene nanoparticles with an Au shell) in reflective mode.[51]

In this case the solution is pumped into the flow cell for real time measurements while a UV-VIS-NIR light sources is shone vertically on the nanosensor surface with the reflected signal detected via a spectrograph with the output extinction spectrum displayed on the laptop screen. ${ }^{[51]}$ In general, the complexity in LSPR technologies mostly relies on the surface of the chip that will be used, while the instrumentation to read the signal is simple. That is in contrast with SPR where its complexity lies mostly on the precise set up to launch a surface plasmon and read it accurately. In LSPR most metals, alloys or semiconductors with a large negative real dielectric constant and small imaginary dielectric constant are theoretically suitable in a number of nanostructured arrangements (Figure 18).

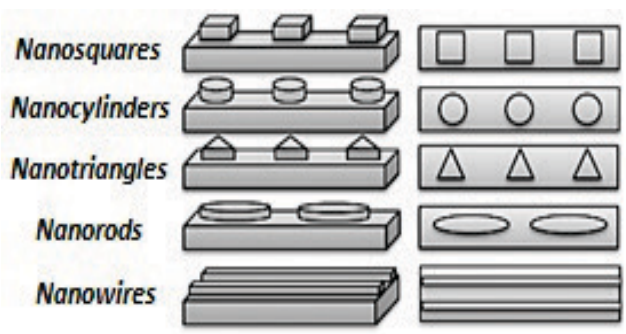

A further example of a commercial LSPR nanobiosensing instrument by LamdaGen including its nanostructured biochip can be seen in Figure 19.

Its operating principle is based on the fact that amongst a broad light spectrum, one wavelength resonantly couples to the localized plasmons on the biochip surface and is absorbed. In particular, the monitored reflected light plasmon has a minimum at this specific wavelength, called $\lambda_{\min }$. This value is dependent on the dielectric constant at the sensor surface. Specifically, changes at the surface as those induced by the binding of biomolecules or other substances, cause changes in the reflecting light including its $\lambda_{\text {min. }}$. These plasmonic changes are the ones precisely monitored via spectroscopy and/or other optical methods including digital ones.

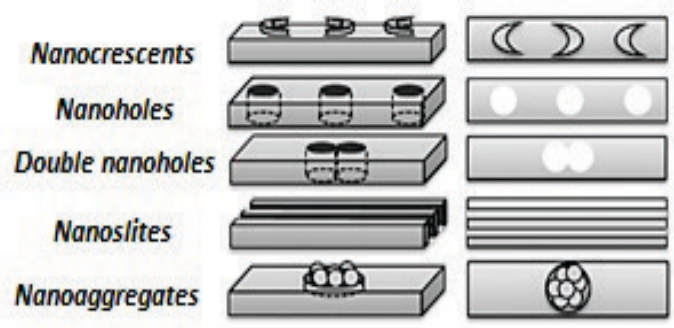

Figure 18. LSPR active substrates: a variety of applicable nanostructures. ${ }^{[52]}$
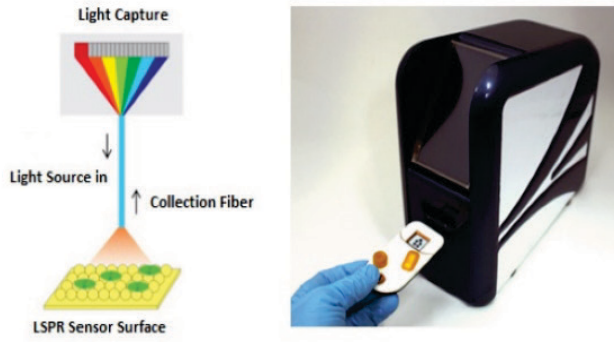

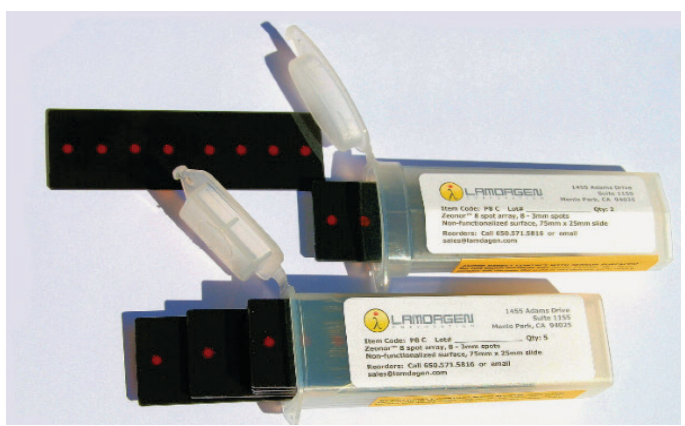

Figure 19. LSPR nanobiosensing. [28] 

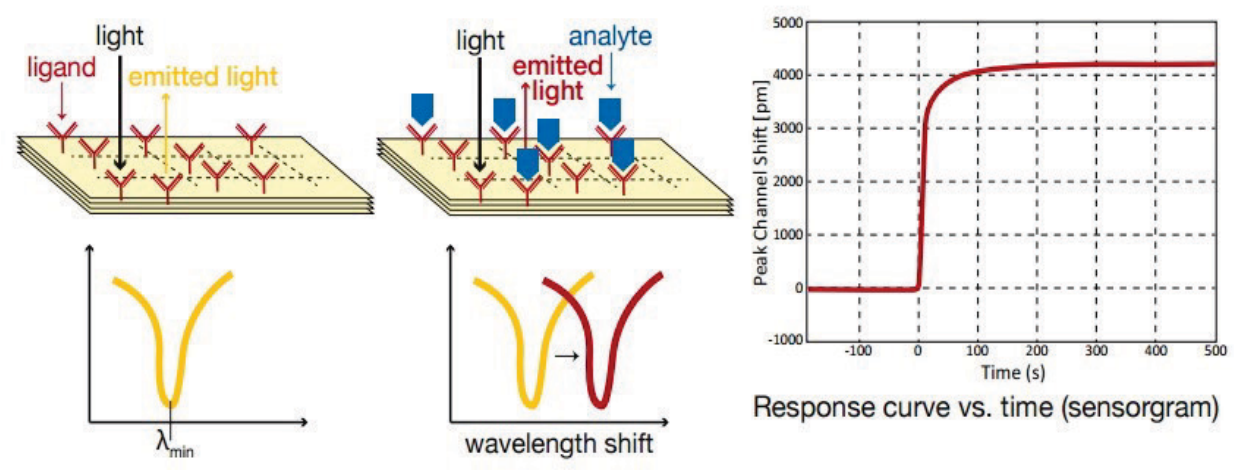

Response curve vs. time (sensorgram)

Figure 20. Real time sensogram in LSPR systems. ${ }^{[28]}$

It is noted that the LamdaGen LSPR biosensor chips integrate billions of $\mathrm{Au}$ nanostructures with reflectivity resembling the UV-Vis of conventional gold colloids (LSPR film has $\lambda_{\max } \sim 550 \mathrm{~nm}$ ) and a refractive index sensitivity $\sim 70-100 \mathrm{~nm} /$ RIU. Upon binding of the target biomolecule on the chip surface there is a shift in the reflected light resulting in real-time sensogram (Figure 20).

Novel designs involving hybrid plasmonic-whispering gallery mode (WGM) nanosensors have also been employed in laboratories settings towards single bioparticle detection due to their enhanced field localization properties. Nevertheless, still some of their drawbacks include long waiting times between bioagent adsorptions as well as first target detection. ${ }^{[53]}$ WGM nanobiosensors are capable of trapping light in their periphery (Figure 21). In this example gold nanoparticles (epitopes) at the equator of a glass microsphere are being used to plasmonically enhance sensing and target analyte capture. In order to achieve resonance a tunable laser light is used through an adjacent fiber. The microsphere resonance excites the plasmon resonance of the gold nanoparticles, which upon target adsorption register a characteristic shift of the resonance frequency.

\section{Self - Assembled, Flexible, One-Dimensional Nanostructures for SERS Applications}

As mentioned previously, what has been widely used in sensors technology is 'LSPR' - localized surface plasmons resonance i.e. collective oscillation of the free electrons of metal nanoparticles resonantly excited by visible light. ${ }^{[56]}$ Nanostructured material, specially designed and roughed surface plated with the metal layer is what provides SERS enhancement. Generally, they can be divided regarding their dimension in noble metal nanoparticles (OD), one dimensional (1D) nanostructures, two dimensional (2D) nanostructures and three dimensional (3D) nanostructures. ${ }^{[57]}$ What plays the key role in all those structures are so-called 'hot-spots', nanogaps between two adjacent nanoparticles with extremely high electromagnetic field enhancement triggered by LSPR. The secret of 'hot spots' is narrow, around $10 \mathrm{~nm}$ gap which can be obtained by selfassembled nanostructures.

Probably, one of the most convincing examples of the flexible nanostructure is given by Schmidt. ${ }^{[58]}$ A onedimensional structure i.e. vertically oriented pillar array, carefully designed regarding surface density, height and

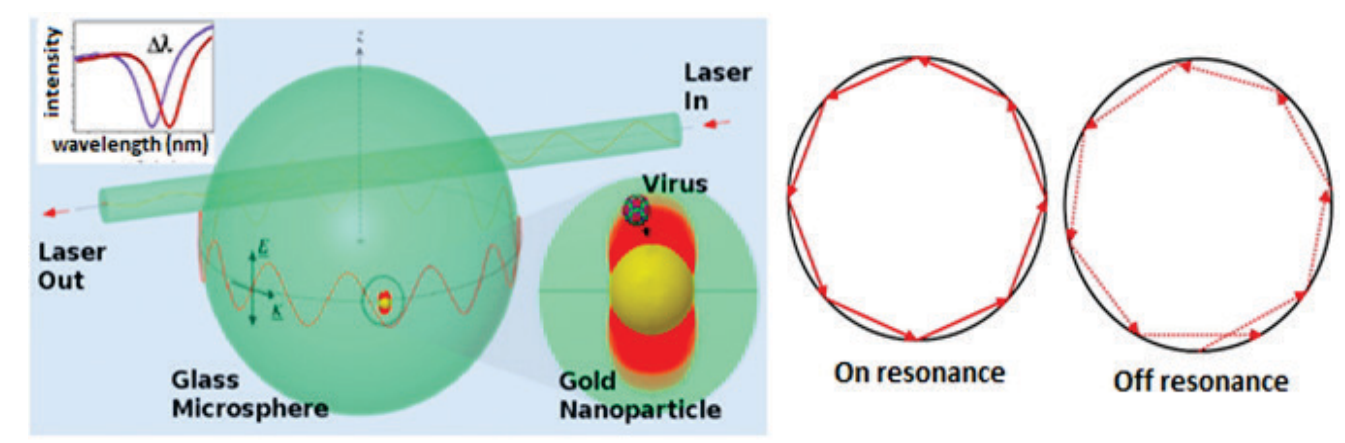

Figure 21. Hybrid plasmonic whispering gallery mode nanobiosensors. ${ }^{[54,55]}$ 

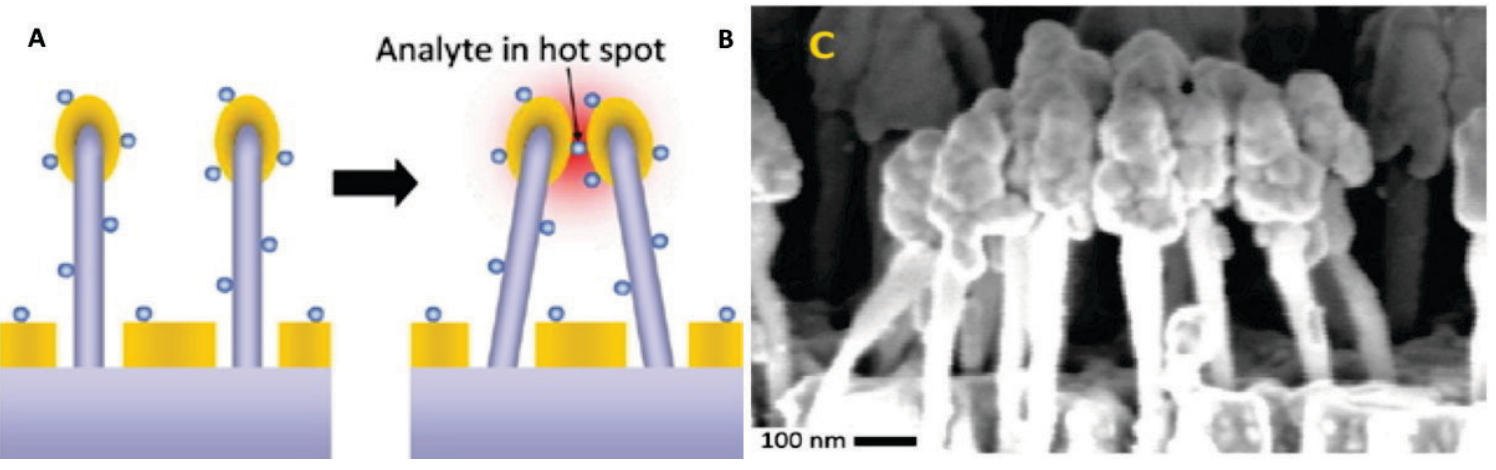

Figure 22. Leaning nanopillars substrate principle: (A) vertically oriented nanopillars before liquid immersion: (B) leaned nanopillars after the immersion; (C) SEM image of the SiNWs after the immersion. ${ }^{[58]}$

shape has a specific aspect ratio (ratio of diameter and length) adequate for bending upon the liquid immersion. Pilar's tips coated with Ag or Ag utilizing evaporation technique leans towards each other's creating pillars bundles (Figure 22). The analyte's molecule trapped between the tips experiences the strong electromagnetic field caused by LSPR which as the consequence results in the significant Raman intensity enhancement. This is the main principle of the solid SERS substrates later explored and developed by Silmeco company.

Spontaneous formation of Raman hot spots created by leaned nanorods gives 100-1000 times higher Raman intensity than the vertical, fixed nanorods. ${ }^{[59]}$ This shows the significance of nanogaps size separation between the metal nanoparticles within a cluster. Except for the geometrical parameters of the nanostructure, the attention should be paid to metal plating technique since it determines the LSPR characteristics. Wu and co. ${ }^{[51]}$ shown that the shape and size of the metal plated tip influences socalled the 'particle mode' giving the contribution to SERS

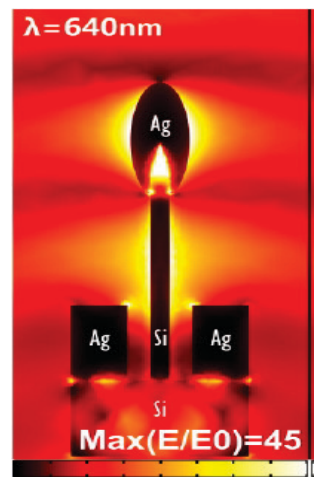

Figure 23. Cross-sectional enhancement factor distributions of an Ag@Si nanopillar with a substrate at its resonant LSP wavelengths. The maximum enhancement factor was based on the electric field on the surface of the nanopillar. Wu et al. ${ }^{[60]}$ enhancement. The other mode can arise from metal covered rod surface, so-called the 'cavity mode'. The resonance position of this mode obviously depends on the silicon pillar thickness. Even more, it is shown that the contribution from the substrate's bottom, for an appropriate pillar height, plated with $\mathrm{Ag}$ or Au can increase the SERS signal (Figure 23).

Similarly, vertically oriented silicon nanowires (SiNWs) can provide strong enhancement factor of $10^{8}$ orders of magnitude. ${ }^{[34]}$ SiNWs have been commonly synthesized through economical Ag-assisted chemical etching technique with hydrofluoric acid. They spontaneously create hot spots as well, however, since the substrate comes in touch with water prior to an analyte, the tweezers like effect does not occur after the analyte introduction (Figure 24).

Another similar example of a vertical silicon nanowire is given in terms of a flexible, gold-coated silicon nanowire with a $1: 10$ aspect ratio.[62] This nanowire is fabricated by the combination of nanosphere lithography and metal-assisted chemical etching. However, this time the 'fingertips' effect is preserved i.e. there is a clear difference between the vertical (dry) and the leaned (after wetting) (Figure 26). The importance of the high aspect ratio is that the aspect ratio of $1: 10$ allows SiNWs bending after the water immersion, the $1: 5$ aspect ratio preserves the vertical SiNWs orientation due to the high stiffness. Interestingly, the author claims that the elastic modulus of

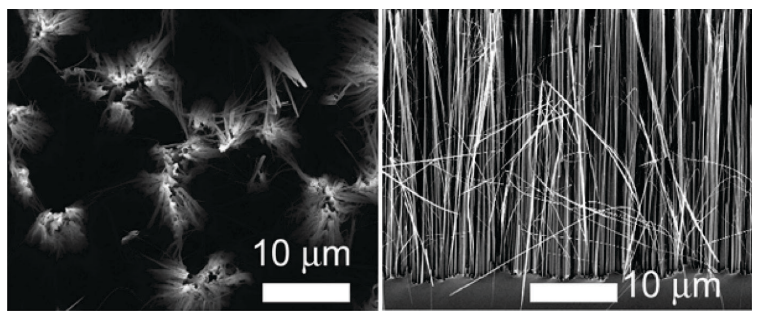

Figure 24. SEM images of top and side view on SiNWs. ${ }^{[61]}$ 
silicon nanowires is independent of their diameters if the diameter is larger than $100 \mathrm{~nm}$. Rather than that, the flexibility of the free-standing silicon nanowires strongly depends on the length of the wires. SiNWs shorter than $0.6 \mu \mathrm{m}$ would have impaired elastic properties and weaker SERS features. As in the previous examples, it is assumed that numerous hot spots were created at the tip-to-tip SiNWs sites.

The 'fingertips' generated hot spots with ultrahigh field enhancement which resulted with melamine limit of detection of $3.2010^{-7} \mathrm{mg} \mathrm{l}^{-1}$. The author demonstrated the importance of angstrom to nanosize fingertips distance for the enhanced SERS signal (Figure 26). Nonleaning wires showed double less intensity than the preleaned wires (brought to lean before the deposition of the analyte) while the pre-leaned wires showed again about double less intensity than post-leaned SiNWs (brought to lean during the deposition of the analyte). This result is in agreement with the results in reference [58].

Besides the vertically oriented pillars and wires, the horizontally oriented, flexible, silicon nanowires (SiNWs) substrates were synthesized by vapour-liquid-solid (VLS) method inside low -pressure chemical vapour deposition (LPCVD) reactor. ${ }^{[63]}$ It is shown that by manipulating the synthetization temperature SiNWs thickness can be reduced to around $50 \mathrm{~nm}$ which together with the length of around $8 \mu \mathrm{m}$, allows flexible nanostructure to occur. The flexible SiNWs creates bundles after the liquid immersion which consequently creates numerous hot spots (Figure 27).

The author has shown the difference in morphology, SERS intensity and lacunarity regarding the two different liquids ethanol (EtOH) and water. SEM images of randomly and horizontally oriented SiNWs for a dry sample and after immersion in $\mathrm{EtOH}$ and water (Figure 28) show the strong difference in morphology between the dry and immersed samples, however, in order to clearly observe the difference between $\mathrm{EtOH}$ and water SERS measurements and lacunarity should be utilized.

Water has higher surface tension and a higher average number of hydrogen bonds than EtOH. The strong adhesive force of water captures SiNWs, pulls them together in bundles and twists during the drying process. This process is significantly stronger in the case of water than EtOH
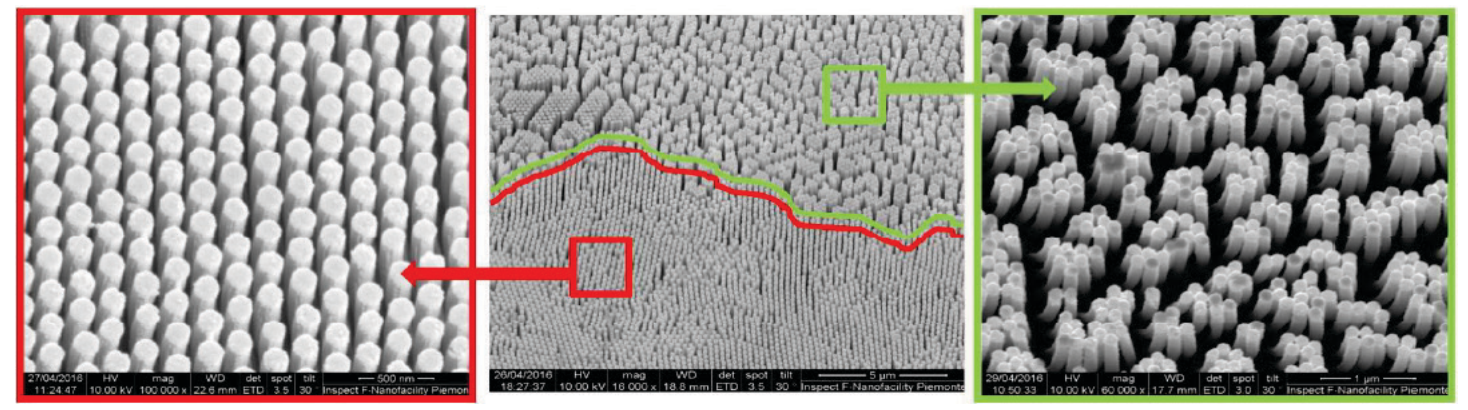

Figure 25. SiNWs obtained by combination of nanospheres lithography and metal-assisted chemical etching (a) vertically oriented SiNWs with aspect ratio $1: 5$ after wetting; (b) morphological difference wet sample with two different aspect ratios $1: 5$ and $1: 10$; (c) morphology of the sample with aspect ratio $1: 10$ after wetting. ${ }^{[62]}$

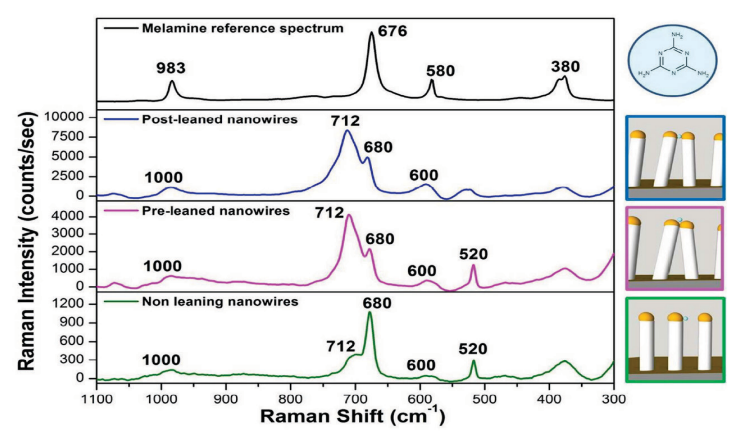

Figure 26. Reference Raman spectrum of melamine powder (black line), post-leaned nanowires (blue line), pre-leaned nanowires (violet line) and non-leaning nanowires (green line). ${ }^{[62]}$

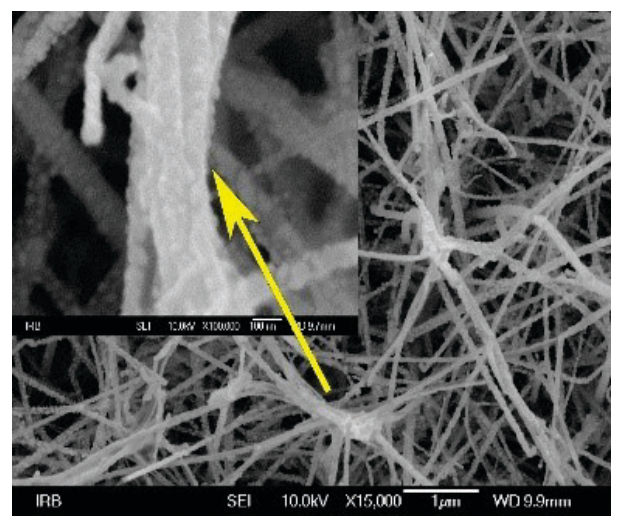

Figure 27. SEM images of SiNWs obtained by VLS process, after the immersion in $\mathrm{H}_{2} \mathrm{O}$. ${ }^{[63]}$ 

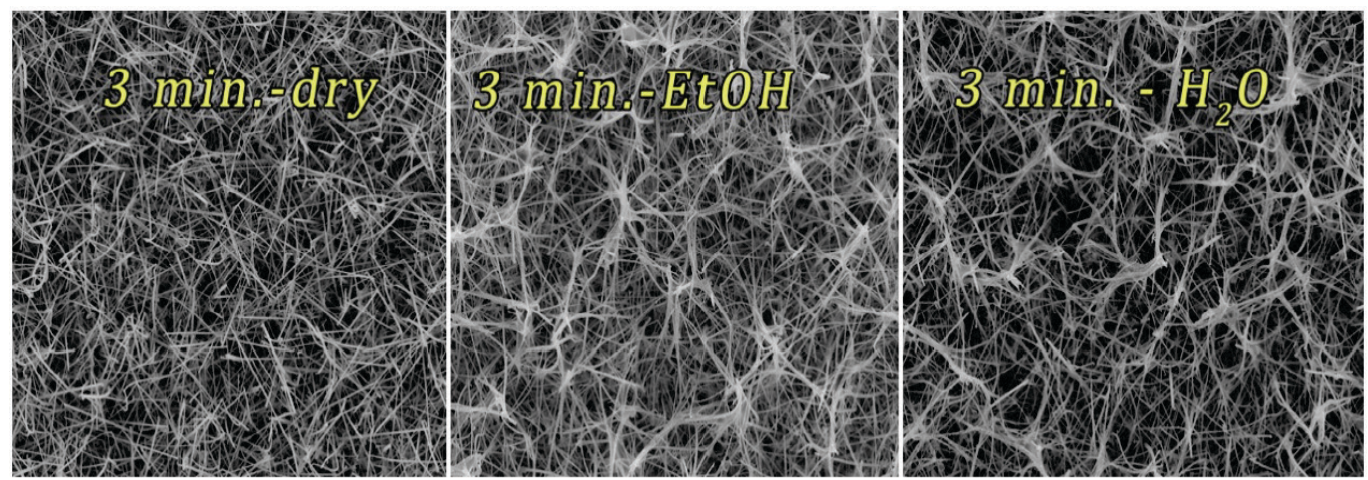

Figure 28. SEM images of randomly and horizontally oriented SiNWs after 3 min Ag sputtering and immersion in EtOH and water. Irregularly shaped SiNWs bundles were created by capillary forces of EtOH and water. ${ }^{[63]}$

and that is the main cause of improved SERS features (Figure 29). In this particular example, the horizontal SiNWs were synthesized at different temperatures from 480 to $560{ }^{\circ} \mathrm{C}$ and were all sputtered with $\mathrm{Ag}$ for $5 \mathrm{~min}$. After that, the samples were dipped in a $10^{-4} \mathrm{M}$ ethanol solution of 4mercaptophenylboronic acid (4-MPBA) for several hours, in order to allow for the formation of SAMs.

Another important factor is plating i.e. the $\mathrm{Ag}$ decoration of SiNWs. Short sputtering time creates $\mathrm{Ag}$ droplets which size defines the position of the plasmon absorption band and consequently influences SERS enhancement. Longer sputtering time covers the SiNWs over the whole length completely which significantly changes the SERS mechanism creating long plated cylinders in charge for the surface nanostructure and hot spots creation. Even longer sputtering time freezes the substrates morphology i.e. after the liquid immersion

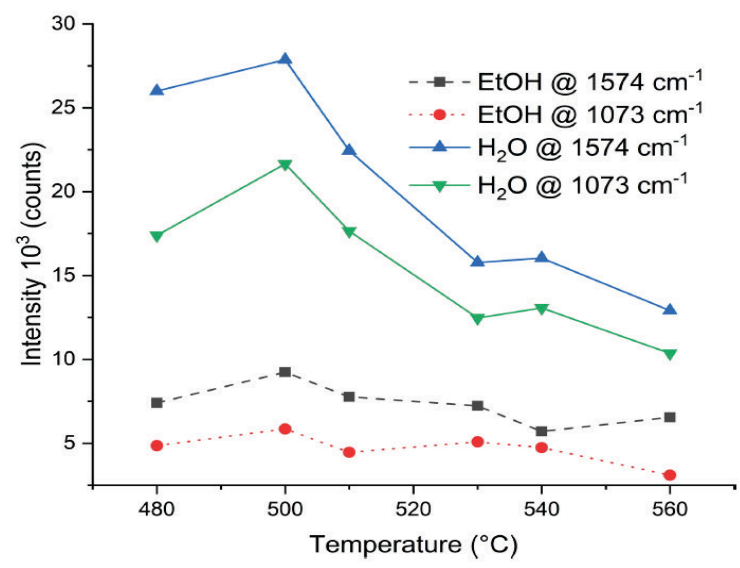

Figure 29. SERS intensities of the 1073 and $1574 \mathrm{~cm}^{-1}$ bands before (denoted EtOH) and after $\mathrm{H} 2 \mathrm{O}$ washing for different VLS process temperatures. The optimal SERS signal is obtained for a temperature of $500{ }^{\circ} \mathrm{C}$ during the VLS process, and the SERS signal significantly increases after washing with water. ${ }^{[63]}$
SiNWs were not flexible since the sputtering fixed the joint points between adjacent SiNWs and the adhesion and surface tension were not strong enough to overcome the stiffening force. This example confirms the importance of metal plating for hot spots occurrence and SERS features. The author concluded that short sputtering time and islandlike $\mathrm{Ag}$ shapes on SiNWs seems to be the best candidates for strong SERS enhancement, however it should be kept in mind that larger analyte concentrations could have beneficial substrate matrix with higher Ag amount since the higher analyte's molecule number demands higher absorption sites on SiNWs.

Fractal dimension and lacunarity are two parameters on which no significant examination has been conducted up to now. Fine tuning of the surface morphology can be examined just by utilizing SEM images and adequate programs for data analyzing. Fractal dimension which can be defined as the measure of complexity i.e. the change in detail with a change in scale showed a clear decrease with immersion in $\mathrm{EtOH}$ and water for short plated samples. Lacunarity is complementary to fractal dimension and can be described as the distribution of the man-sized gaps between SiNWs i.e. the measure of heterogeneity (inhomogeneity) or translational-rotational invariance in an image. Following the results from the fractal dimension analysis, lacunarity has shown the difference between $\mathrm{EtOH}$ and water immersed samples for shortly plated samples as well. In this way, by implementing the fractal and lacunar analysis, a limit for flexible SiNWS to occur is obtained. It is shown that SiNWs should not be thicker than $120 \mathrm{~nm}$ after $\mathrm{Ag}$ plating in order to preserve flexibility and tweezers like effect. ${ }^{[63]}$

In conclusion, one dimensional, flexible nanostructure such as SiNWs has shown the advanced SERS features over the standard substrates with the fixed morphologies. The structural flexibility can be achieved through a certain aspect ratio and adequate metal plating. It is indicated that a high aspect ratio and decoration with metal nanoparticles 

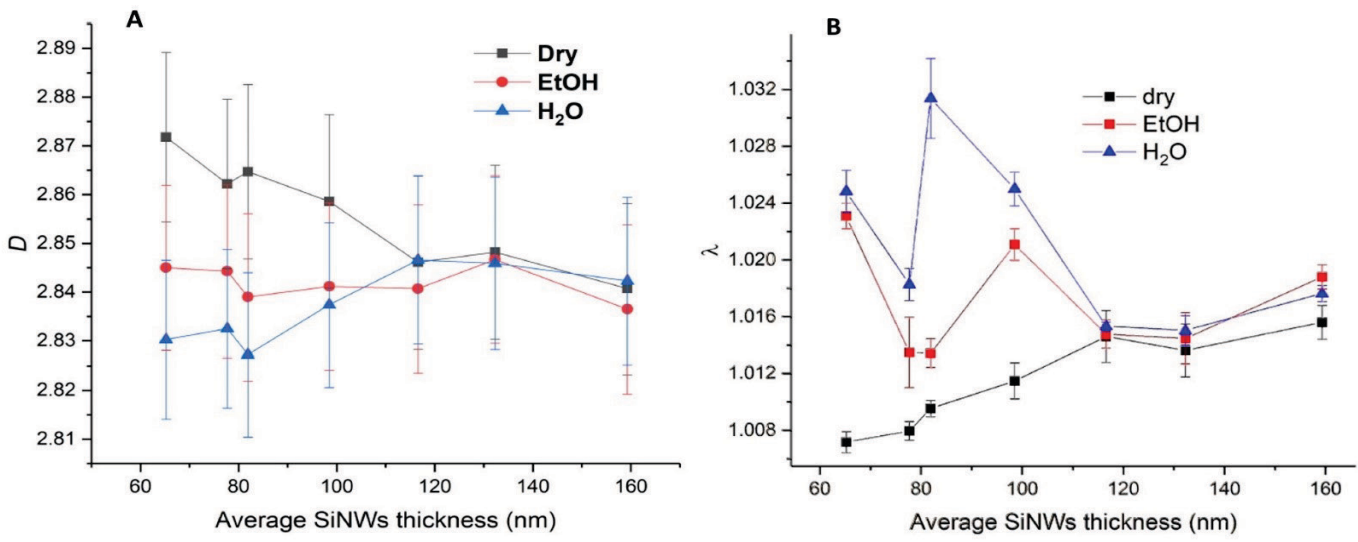

Figure 30. (A) fractal dimension; (B) lacunarity vs. average SiNWS thickness. [63]

are beneficial for such flexible substrates. The main contribution to SERS mechanism these samples accomplish through narrowing the gaps between the metal nanoparticles which consequently enhances the electric field and SERS. Presented works encourage the fabrication of flexible SERS substrates with different morphologies than nanopillars and nanowires.

\section{Approaches in SERS Detection: Oriented and Random Configurations as well as Direct and Indirect Routes}

As discussed, the use of metallic nanoparticles can lead to SERS which can yield results both in random and oriented configurations, with each approach exhibiting its own advantages. In particular SERS can be used in random configuration measurements performed in a simple and fast way by dropping a molecule of solution to be adsorbed on the SERS substrate. Nevertheless, despite their simplicity and speed the main limitation of such measurements is that they are non-reproducible as the target molecule adsorption is not controlled and more specifically its orientation. Furthermore, the direct contact of the biological molecules with the metal may destroy them. As a result, the preferred method is the oriented SERS configuration where the requirement is the substrate surface functionalization which

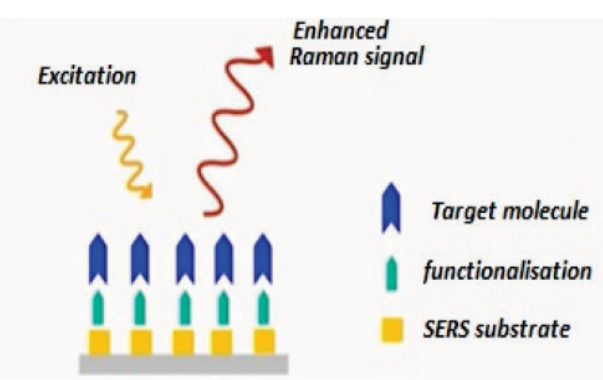

Figure 31. Oriented SERS configuration. ${ }^{[64]}$ helps in better fixing and orientating the target molecule leading to reproducible SERS signals. Despite the reproducibility in signal and added specificity in target molecule detection care needs to be exercised when performing SERS that the spacers used are not too long such as the target molecule is not too far from the nanoparticle (substrate) and the SERS effect becomes limited or decreased (Figure 31).

There is an instrumental set up using inverted dark field microscopy in order to determine SERS signals and resonance peak shifts (LSPR) as introduced by Portella et.al.[65] (Figure 32). They have used a microfluidic chip on a piezo stage while they prepared biochips for target molecule attachment taking care of the fact that a reduced gap in their nanoantennas would cause an increase of the near field intensity. Any changes in the refractive index upon molecular binding would lead to shifts in the resonance frequency.

Furthermore, the SERS detection of specific biomolecules can be either accomplished directly as discussed above or indirectly. The later usually involves the association of a Raman active moiety, e.g. a fluorescent dye attached to the target molecule. In Figure 33 the cross-

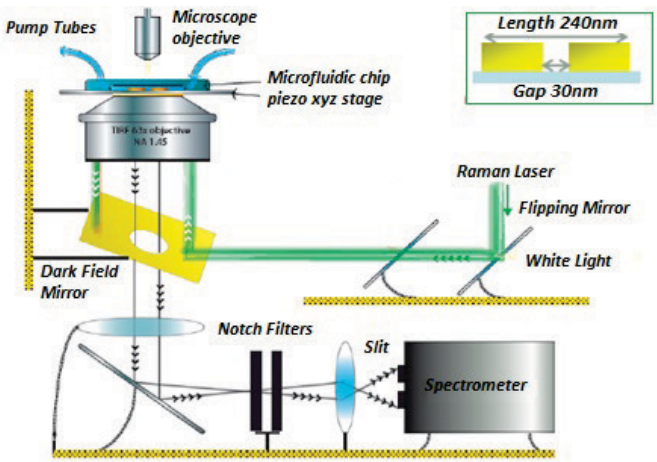

Figure 32. Biosensing using SERS and LSPR. ${ }^{[65]}$ 


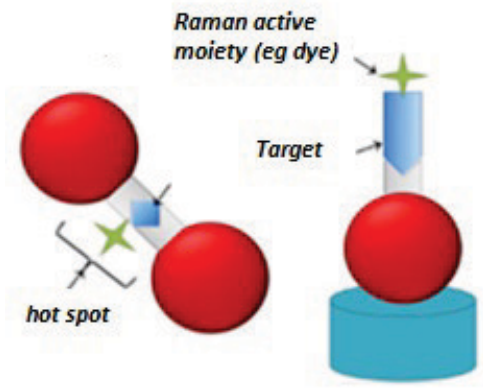

Figure 33. Cross linking strategies using Raman-active moieties leading to enhanced SERS signals. [66]

linking approach is displayed where upon target detection the Raman dye is entrapped in a 'hot-spot', i.e. in this case between two nanoparticles leading to further enhancement of the Raman signal.

\section{CONCLUSION}

It is evident that recent advances in nanotechnology have had a substantial impact in plasmonic nanobiosensing leading from the more conventional SPR biosensors which have been around since the 1990's to novel LSPR designs based on the properties of metallic nanoparticles. Such LSPR sensors offer significant advances in signal sensitivity as well as allowing for the development of single molecule detection protocols. Their simplicity in design allows for production with lower costs as well as potentially leading to successful in vivo applications where they can probe single cells for the detection of various biomarkers as well as the presence of single viruses. Towards this direction a significant amount of research is directed in order to allow for low cost devices with high sensitivity and short response time. Furthermore, the use of nanosubstrates for LSPR allows at the same time significant Raman signal enhancement which in turn can be utilized for the exact determination of biomolecules based on their vibrational spectra detecting each time the relevant Stokes shifts.

Acknowledgment. The authors would like to thank the Bilateral Program for Academic Mobility, Republic of Croatia for their support of this work via Scholarship Number 60403/18-02/00006, 533-10-180-0001 as well as the SAFU project KK.01.1.1.01.0001 and the Croatian Science Foundation (project IP-2014-09-7046).

\section{REFERENCES}

[1] J. E. N. Dolatabadi, O. Mashinchian, B. Ayoubi, A. A. Jamali, A. Mobed, D. Losic, Y. Omidi, M. D. L. Guardia, Trends Anal. Chem. 2011, 30, 459-472. https://doi.org/10.1016/j.trac.2010.11.010
[2] A. Touhami in nanomedicine, (Eds.: A. Seifalian, A. D. Mel, D. M. Kalaskar), One Central Press, 2014, pp. 374-403.

[3] A. Chamorro-Garcia, A. Merkoçi, Nanobiomedicine (Rij). 2016, 3, 1849543516663574. https://doi.org/10.1177/1849543516663574

[4] N. Bhalla, P. Jolly, N. Formisano, P. Estrela, Essays Biochem. 2016, 60, 1-8. https://doi.org/10.1042/EBC20150001

[5] D. W. G. Morrisson, M. R. Dokmeci, U.Demirci in Biomedical nanostructures, (Eds.: K. Gonsalves, C. Halberstadt, C. T. Laurencin, L. Nair), John Wiley \& Sons, 2007, pp. 433-455.

[6] S. Neethirajan, V. Ragavan, X. Weng, R. Chand, Biosensors. 2018, 8, 1-34. https://doi.org/10.3390/bios8010023

[7] H. Kaura, M. Shorie, Nanoscale Adv. 2019,1, 2123 2138. https://doi.org/10.1039/C9NA00153K

[8] L. Tong, Sensors. 2018, 18, 1-27. https://doi.org/10.3390/s18030903

[9] P. M. Kasili, PhD dissertation, University of Tennessee Knoxville, US, 2004.

[10] T. Vo-Dinh, Protein Nanotechnology. 2005, 300, 383401. https://doi.org/10.1385/1592598587

[11] Y. Zhang, T. H. Wang, Theranostics 2012, 2, 631-654. https://doi.org/10.7150/thno.4308

[12] Q. Chen, A. Kiraz, X. Fan, Lab Chip. 2016, 16, 353359. https://doi.org/10.1039/C5LC01004G

[13] C. Y. Zhang, H. C. Yeh, M. T. Kuroki et al., Nature Materials 2005, 4, 826-831.

https://doi.org/10.1038/nmat1508

[14] T. Vo-Dinh, P. Kasili, M. Wabuyele, Nanomedicine 2006, 2, 22-30.

https://doi.org/10.1016/j.nano.2005.10.012

[15] A. J. Haes, C. L. Haynes, A. D. McFarland, G. C. Schatz, R. P. Van Duyne, S. Zou, MRS Bulletin.2005, 30, 368375. https://doi.org/10.1557/mrs2005.100

[16] K. A. Willets, R. P. Van Duyne, Annu Rev Phys Chem. 2007, 58, 267-297.

https://doi.org/10.1146/annurev.physchem.58.032 806.104607

[17] S. Unser, I. Bruzas, J. He, L. Sagle, Sensors. 2015, 15, 15684-15716. https://doi.org/10.3390/s150715684

[18] T. Rojalin, Br. Phong, H. J. Koster, R. P. Carney, Front Chem. 2019, 7, 279.

https://doi.org/10.3389/fchem.2019.00279

[19] B. Liedberg, C. Nylander, I. Lundstrom, Sensors and Actuators 1983, 4, 299-304. https://doi.org/10.1016/0250-6874(83)85036-7

[20] E. Kretschmann, Z. Phys. 1971, 241, 313-324. https://doi.org/10.1007/BF01395428

[21] B. A. Prabowo, A. Purwidyantri, K. Liu, Biosensors 2018, 8, 80-107. 
https://doi.org/10.3390/bios8030080

[22] Y. H. Ichikawa, Progress of Theoretical Physic. 1957, 18, 247-263.

https://doi.org/10.1143/PTP.18.247

[23] S. Basu, P. Sen, Advances in Quantum Chemistry 1972, 6, 159-175.

https://doi.org/10.1016/S0065-3276(08)60544-4

[24] J. J. Monaghan, Aust. J. Phys. 1973, 26, 597-606. https://doi.org/10.1071/PH730597

[25] Y. Kornyushin, M. Y. Amusia, Contemporary Physics. 2001, 42, 159-165. https://doi.org/10.1080/00107510110048057

[26] J. M. Pitarke, V. M. Silkin, E. V. Chulkov, P. M. Echenique, Rep. Prog. Phys. 2007, 70, 1-87. https://doi.org/10.1088/0034-4885/70/1/R01

[27] Nano Optics Course, ETHZ, Switzerland. 2016 https://www.photonics.ethz.ch/fileadmin/user_upl oad/Courses/NanoOptics/plasmons2.pdf (accessed 21 July 2019).

[28] Lamdagen. 2016, http://lamdagen.com/wp-content/ uploads/2014/03/lg-Ispr-sensors-vs-au-colloids.pdf (Accessed 21 July 2019)

[29] O. Benson, Nature 2011, 480, 193-199. https://doi.org/10.1038/nature10610

[30] Park Lab 2016, http://ecee.colorado.edu/ wpark/ research.html (Accessed 21 July 2019).

[31] E. Petryayeva, U. J. Krull, Anal. Chim. Acta 2011, 706, 8-24. https://doi.org/10.1016/j.aca.2011.08.020

[32] M. Wang, M. Ye. J. locozzia, C. Lin, Z. Lin, Adv. Sci. 2016, 3, 1600024.

https://doi.org/10.1002/advs.201600024

[33] X. Huang, M. A. El-Sayeda, J. Advanced Research. 2010, 1, 13-28.

https://doi.org/10.1016/j.jare.2010.02.002

[34] E. Martinson, Department of Physics, Chemistry and Biology (IFM) 2015, https://www.ifm.liu.se/applphys/ molphys/research/biosensing_using_nanopart/ (accessed 21 July 2019).

[35] I. Choi, Y. Choi, IEEE J. Sel. Top. Quantum Electron. 2012, 18, 1110-1121. https://doi.org/10.1109/JSTQE.2011.2163386

[36] A. Haes, R. Van Duyne, Anal Bioanal Chem. 2004, 379, 920-930.

https://doi.org/10.1007/s00216-004-2708-9

[37] C. David, N. Guillot, H. Shen, T. Toury ,M. L. de la Chapelle, Nanotechnology 2010, 21, 475501. https://doi.org/10.1088/0957-4484/21/47/475501

[38] I. Bruzas, W. Lum, Z. Gorunmez, L. Sagle, Analyst. 2018, 143, 3990-4008.

https://doi.org/10.1039/C8AN00606G

[39] B. Sharma, R. R. Frontiera, A. I. Henry, E. Ringe, R. P. Van Duyne, Mater. Today. 2012, 15, 16-25. https://doi.org/10.1016/S1369-7021(12)70017-2
[40] X. Tang, W. Cai, L. Yang, J. Liu, 2013, Nanoscale 5, 11193-11199. https://doi.org/10.1039/c3nr03671e

[41] N. Guillot, M. L. de la Chapelle, M. Lamy, J Quant Spectrosc Ra. 2012, 113, 2321-2333. https://doi.org/10.1016/j.jqsrt.2012.04.025

[42] U. Dinish, F. C. Yaw, A. Agarwal, M. Olivo, Biosens. Bioelectron. 2011, 26, 1987-1992. https://doi.org/10.1016/j.bios.2010.08.069

[43] L. Mikac, M. Ivanda, V. Derek, M. Gotić, J. Raman Spectrosc. 2016, 47, 1036-1041. https://doi.org/10.1002/jrs.4911

[44] L. Mikac, M. Ivanda, M. Gotic, T. Miheli, L. Horvat, J Nanopart Res.2014, 16, 2748-2751. https://doi.org/10.1007/s11051-014-2748-9

[45] T. Janči, L. Mikac, M. Ivanda, N. M. Radovčić, H. Medić, S. Vidaček, J. Raman Spectrosc. 2017, 48, 6472. https://doi.org/10.1002/jrs.4991

[46] T. Janči, D. Valinger, J.G. Kljusurić, L. Mikac, S. Vidaček, M. Ivanda, Food Chem. 2017, 224, 48-54. https://doi.org/10.1016/j.foodchem.2016.12.032

[47] S. Lofas, B. A. Johnsson, J. Chem. Soc. Chem. Commun. 1990, 21, 1526-1528. https://doi.org/10.1039/C39900001526

[48] S. Lofas, Pure \& Appl.Chem. 1995, 67, 829-834. https://doi.org/10.1351/pac199567050829

[49] Biacore X100, Label-free interaction analysis equipment developed by General Electrics, GE, USA.

[50] Sensor Chip CM5 for the Biacor X100 equipment, developed by General Electrics, GE, USA.

[51] S. Yang, T. Wu, X. Zhao, W. Tan, Sensors. 2014, 14, 13273-13284. https://doi.org/10.3390/s140713273

[52] N. Guillot, M. L. de la Chapelle, J. Nanophotonics 2012, 6, 064506. https://doi.org/10.1117/1.JNP.6.064506

[53] S. L. Shopova, R. Rajmangal, S. Holler, S. Arnold, Appl. Phys. Lett. 2011, 98, 243104. https://doi.org/10.1063/1.3599584

[54] V. R. Dantham, S. Holler, V. Kolchenko, Z. Wan, S. Arnold, Appl. Phys. Lett. 2012, 101, 043704. https://doi.org/10.1063/1.4739473

[55] J. M. Gamba, PhD Thesis, California Institute of Technology, US, 2012

[56] A. Urrutia, J. Goicoechea, F. J. Arregui, J. Sensors, 2015, 805053. https://doi.org/10.1155/2015/805053

[57] X. Chen, C. Jiang, S. Yu, CrystEngComm. 2014, 16, 9959-9973. https://doi.org/10.1039/C4CE01383B

[58] M. S. Schmidt, J. Hübner, A. Boisen, Adv. Opt. Mater. 2012, 24, OP11-OP18. https://doi.org/10.1002/adma.201103496

[59] H. Liu, Y. Sun, Z. Jin, L. Yang, J. Liu, Chem. Sci. RSC. 2013, 4, 3490-3496. https://doi.org/10.1039/c3sc51231b 
[60] K. Wu, T. Rindzevicius, M. S. Schmidt, K. B. Mogensen, S. Xiao, A. Boisen, Optics Express. 2015, 23, 12965-12978.

https://doi.org/10.1364/OE.23.012965

[61] M. L. Zhang, X. Fan, H. W. Zhou, M. W. Shao, J. A. Zapien, N. B. Wong, S. T. Lee, J. Phys. Chem. C. 2010, 114, 1969-1975.

https://doi.org/10.1021/jp902775t

[62] S. A. Kara, A. Keffous, A. M. Giovannozzi, A. M. Rossi, E. Cara, L. D.'Ortenzi, K. Sparnacci, L. Boarino, N. Gabouze, S. Soukanea, RSC Advances 2016, 6, 93649-93659.

https://doi.org/10.1039/C6RA20323J
[63] H. Gebavi, V, Gašparić, D. Risović, N. Baran, P. H. Albrycht, M. Ivanda, J. Nanotechnol. 2019, 10, 725734. https://doi.org/10.3762/bjnano.10.72

[64] P. Rostron, D. Gerber, Int. J. Eng. Technical Res. 2016, 6, 50-64.

[65] C. Portella, Seminario Nanotecnologia Cuba 2012, http://www.seminarionanotecnologiascuba.com/Me moriasIV/pdf/Design\%20and\%20Nanofabrication\%2 0of\%20a\%20Biosensor.pdf (Accessed 8 April 2016).

[66] G. Doria, J. Conde, B. Veigas, L. Giestas, C. Almeida, M. Assunção, J. Rosa J, P.V .Baptista, Sensors 2012, 12, 1657-1687.

https://doi.org/10.3390/s120201657 